H.X. YI

KODAI MATH. J.

13 (1990), 363-372

\title{
MEROMORPHIC FUNCTIONS THAT SHARE TWO OR THREE VALUES
}

\author{
By HoNG-XuN YI
}

\section{Introduction and Main Results.}

Let $f(z)$ and $g(z)$ be two nonconstant meromorphic functions in the complex plane. If $f$ and $g$ have the same $a$-points with the same multiplicities, we say $f$ and $g$ share the value a $C M$. (see [2]). It is assumed that the reader is familiar with the fundamental concepts of Nevanlinna's theory of meromorphic functions and their standard symbols, as found in [3]. It will be convenient to let $E$ denote any set of finite linear measure of $0<r<\infty$ and let $I$ denote any set of infinite linear measure of $0<r<\infty$. The notation $S(r, f)$ denotes any quantity satisfying $S(r, f)=o(T(r, f))(r \rightarrow \infty, r \notin E)$.

M. Ozawa proved the following result.

THEOREM A (see [5]). Let $f$ and $g$ be entire functions of finite order such that $f$ and $g$ share $0,1 C M$. If $\delta(0, f)>1 / 2$, then $f \cdot g=1$ unless $f=g$.

In [9] H. Ueda showed that in Theorem $A$ the order restriction of $f$ and $g$ can be removed. He proved more generally the following result.

THEOREM B. Let $f$ and $g$ be meromorphic functions such that $f$ and $g$ share $0,1, \infty C M$. If

$$
\lim _{r \rightarrow \infty} \sup \frac{N(r, 1 / f)+N(r, f)}{T(r, f)}<\frac{1}{2}
$$

then $f=g$ or $f \cdot g=1$.

Recently the present author proved the following result.

THEOREM C (see [13]). Let $f$ and $g$ be meromorphic functions such that $f$ and $g$ share $0,1, \infty C M$. If

$$
\bar{N}\left(r, \frac{1}{f}\right)+\bar{N}(r, f)<(\lambda+o(1)) T(r, f) \quad(r \in I),
$$

where $\lambda<1 / 2$, then $f=g$ or $f \cdot g=1$.

Received July 3, 1989; Revised March 12, 1990. 
In order to state our first theorem, we introduce the following notations.

Let $f(z)$ be a meromorphic function. We denote by $n_{1}(r, 1 / f)$ the number of simple zeros of $f$ in $|z| \leqq r$ and by $n_{1}(r, f)$ the number of simple poles of $f$ in $|z| \leqq r . \quad N_{1}(r, 1 / f)$ and $N_{1}(r, f)$ are defined in terms of $n_{1}(r, 1 / f)$ and $n_{1}(r, f)$ respectively in the usual way.

Let $f(z)$ and $g(z)$ be meromorphic functions. We denote by $T(r)$ the maximum of $T(r, f)$ and $T(r, g)$.

In this paper we prove the following result which is an improvement of the above results.

THEOREM 1. Let $f$ and $g$ be meromorphic functions such that $f$ and $g$ share $0,1, \infty C M$. If

$$
N_{1}\left(r, \frac{1}{f}\right)+N_{1}(r, f)<(\lambda+o(1)) T(r) \quad(r \in I),
$$

where $\lambda<1 / 2$, then $f=g$ or $f \cdot g=1$.

By Theorem 1 we immediately obtain the following corollary.

COROLLARY 1. Let $f$ and $g$ be meromorphic function such that $f$ and $g$ share $0,1, \infty C M$. If

$$
\lim _{r \rightarrow \infty} \sup \frac{N_{1}(r, 1 / f)+N_{1}(r, f)}{T(r)}<\frac{1}{2},
$$

then $f=g$ or $f \cdot g=1$.

In [7] $\mathrm{H}$. Ueda proved the following result.

THEOREM D. Let $f$ and $g$ be entire functions such that $f$ and $g$ share 0,1 $C M$. If all zero-points of $f$ excepting at most finite number have multiplicities $\geqq 2$, then $f=g$ or $f \cdot g=1$.

From Theorem 1 we immediately deduce the following corollary which is an improvement of Theorem $\mathrm{D}$.

COROLLARY 2. Let $f$ and $g$ be meromorphic functions such that $f$ and $g$ share $0,1, \infty C M$. If all zero-points and pole-points of $f$ excepting at most finite number have multiplicities $\geqq 2$, then $f=g$ or $f \cdot g=1$.

In [5] M. Ozawa proved the following theorem.

THEOREM E. Let $f$ and $g$ be entire functions such that $f$ and $g$ share $1 C M$. If $\delta(0, f)>0$ and 0 is lacunary for $g$, then $f=g$ or $f \cdot g=1$.

Recently the present author proved the following result which is an extension of Theorem $\mathrm{E}$. 
THEOREM F (see [11]). Let $f$ and $g$ be meromorphic functions such that $f$ and $g$ share $1 C M$. If $\delta(0, f)+\delta(0, g)>1$ and $\delta(\infty, f)=\delta(\infty, g)=1$, then $f=g$ or $f \cdot g=1$.

In this paper we prove the following result which is an improvement of the above theorems.

THEOREM 2. Let $f$ and $g$ be meromorphic functions such that $f$ and $g$ share $1, \infty C M$. If

$$
N\left(r, \frac{1}{f}\right)+N\left(r, \frac{1}{g}\right)+2 \bar{N}(r, f)<(\mu+o(1)) T(r) \quad(r \in I),
$$

where $\mu<1$, then $f=g$ or $f \cdot g=1$.

By Theorem 2 we immediately obtain the following corollary.

COROLLARY 3. Let $f$ and $g$ be meromorphic functions such that $f$ and $g$ share $1, \infty C M$. If $\delta(0, f)+\delta(0, g)+2 \Theta(\infty, f)>3$, then $f=g$ or $f \cdot g=1$.

Let $f(z)=2 e^{z}\left(1-2 e^{z}\right), g(z)=(1 / 4) e^{-z}\left(2-e^{-z}\right)$. It is easy to see that this example shows that the theorems and corollaries in this paper are sharp.

\section{Some Lemmas.}

The following lemmas will be needed in the proof of our theorems.

LEMMA 1. Let $f$ and $g$ be two nonconstant meromorphic functions, and let $c_{1}, c_{2}$ and $c_{3}$ be three nonzero constants. If $c_{1} f+c_{2} g=c_{3}$, then

$$
T(r, f)<N\left(r, \frac{1}{f}\right)+N\left(r, \frac{1}{g}\right)+\bar{N}(r, f)+S(r, f) .
$$

Proof. By the second fundamental theorem, we have

$$
\begin{aligned}
T(r, f) & <N\left(r, \frac{1}{f}\right)+N\left(r,\left(f-\frac{c_{3}}{c_{1}}\right)^{-1}\right)+\bar{N}(r, f)+S(r, f) \\
= & N\left(r, \frac{1}{f}\right)+N\left(r, \frac{1}{g}\right)+\bar{N}(r, f)+S(r, f),
\end{aligned}
$$

which proves Lemma 1.

LEMMA 2 (see [4]). Let $f_{1}, f_{2}, \cdots, f_{n}$ be linearly independent meromorphic functions satisfying $\sum_{i=1}^{n} f_{\imath}=1$. Then for $j=1,2, \cdots, n$, we have 


$$
\begin{gathered}
T\left(r, f_{\jmath}\right)<\sum_{\imath=1}^{n} N\left(r, \frac{1}{f_{i}}\right)+N\left(r, f_{\jmath}\right)+N(r, D)-\sum_{\imath=1}^{n} N\left(r, f_{\imath}\right) \\
-N\left(r, \frac{1}{D}\right)+O\left(\log r+\log T_{n}(r)\right) \quad(r \notin E),
\end{gathered}
$$

where $D$ denotes the Wronskian

$$
D=\left|\begin{array}{c}
f_{1} \quad, f_{2} \quad, \cdots, f_{n} \\
f_{1}^{\prime}, f_{2}^{\prime} \quad, \cdots, f_{n}^{\prime} \\
\cdots \cdots \cdots \cdots \cdots \cdots \cdots \cdots \cdots \cdots . . . \cdots \\
f_{1}^{(n-1)}, f_{2}^{(n-1)}, \cdots, f_{n}^{(n-1)}
\end{array}\right|
$$

and $T_{n}(r)$ denotes the maximum of $T\left(r, f_{\imath}\right), i=1,2, \cdots, n$.

LEMMA 3. Let $f_{1}, f_{2}$ and $f_{3}$ be three nonconstant meromorphic functions satisfying $\sum_{\imath=1}^{3} f_{2}=1$, and let $g_{1}=-f_{3} / f_{2}, g_{2}=1 / f_{2}, g_{3}=-f_{1} / f_{2}$. If $f_{1}, f_{2}$ and $f_{3}$ are linearly independent, then $g_{1}, g_{2}$ and $g_{3}$ are linearly independent.

Proof. Suppose that $g_{1}, g_{2}$ and $g_{3}$ are linearly dependent. Then there exist three constants $\left(c_{1}, c_{2}, c_{3}\right) \neq(0,0,0)$ such that

$$
c_{1} g_{1}+c_{2} g_{2}+c_{3} g_{3}=0,
$$

that is

$$
c_{1} f_{3}+c_{3} f_{1}=c_{2} \text {. }
$$

If $c_{2}=0$, then $c_{1} \neq 0, c_{3} \neq 0$, and

$$
c_{1} f_{3}+c_{3} f_{1}=0,
$$

which contradicts our assumption.

If $c_{2} \neq 0$, from (3) we have

$$
\frac{c_{1}}{c_{2}} f_{3}+\frac{c_{3}}{c_{2}} f_{1}=1 .
$$

Noting $\sum_{i=1}^{3} f_{\imath}=1$, from (4) we get

$$
\left(1-\frac{c_{3}}{c_{2}}\right) f_{1}+f_{2}+\left(1-\frac{c_{1}}{c_{2}}\right) f_{3}=0 .
$$

which is impossible.

This completes the proof of Lemma 3 .

LEMMA 4. Let $h(z)$ be a nonconstant entire function. Then

$$
T\left(r, h^{\prime}\right)=o\left(T\left(r, e^{h}\right)\right) \quad(r \notin E) .
$$


Proof. We have

$$
T\left(r, h^{\prime}\right) \leqq T(r, h)+S(r, h) .
$$

On other hand, by Clunie's result (see, [3, pp 54]), we have

Thus

$$
T(r, h)=o\left(T\left(r, e^{h}\right)\right) .
$$

$$
T\left(r, h^{\prime}\right)=o\left(T\left(r, e^{h}\right)\right) \quad(r \notin E),
$$

which proves Lemma 4.

\section{Proof of Theorem 2.}

By assumption, we have

$$
f-1=e^{h}(g-1) \text {, }
$$

where $h$ is an entire function. Let $f_{1}=f, f_{2}=e^{h}, f_{3}=-e^{h} g$ and $T_{3}(r)$ denote the maximum of $T\left(r, f_{2}\right), i=1,2,3$. From (5) we have

$$
\begin{aligned}
& \sum_{\imath=1}^{3} f_{\imath}=1, \\
& \sum_{\imath=1}^{3} N\left(r, \frac{1}{f_{i}}\right)=N\left(r, \frac{1}{f}\right)+N\left(r, \frac{1}{g}\right),
\end{aligned}
$$

and

$$
T_{3}(r)=O(T(r)) .
$$

We discuss the following two cases.

a) Suppose that $f_{1}, f_{2}$ and $f_{3}$ are linearly independent. By Lemma 2 and (8), we have

$$
T(r, f)<\sum_{\imath=1}^{3} N\left(r, \frac{1}{f_{i}}\right)+N(r, D)-N\left(r, f_{2}\right)-N\left(r, f_{3}\right)+o(T(r)) \quad(r \notin E),
$$

where

$$
D=\left|\begin{array}{c}
f_{1}, f_{2}, f_{3} \\
f_{1}^{\prime}, f_{2}^{\prime}, f_{3}^{\prime} \\
f_{1}^{\prime \prime}, f_{2}^{\prime \prime}, f_{3}^{\prime \prime}
\end{array}\right| .
$$

From (6) and (10) we get

$$
D=\left|\begin{array}{c}
f_{2}^{\prime}, f_{3}^{\prime} \\
f_{2}^{\prime \prime}, f_{3}^{\prime \prime}
\end{array}\right|
$$

and hence

$$
N(r, D)-N\left(r, f_{2}\right)-N\left(r, f_{3}\right) \leqq N\left(r, g^{\prime \prime}\right)-N(r, g)=2 \bar{N}(r, g)=2 \bar{N}(r, f) .
$$

From (7), (9) and (11) we obtain 


$$
T(r, f)<N\left(r, \frac{1}{f}\right)+N\left(r, \frac{1}{g}\right)+2 \bar{N}(r, f)+o(T(r)) \quad(r \notin E) .
$$

Let $g_{1}=-f_{3} / f_{2}=g, g_{2}=1 / f_{2}=e^{-h}, g_{3}=-f_{1} / f_{2}=-e^{-h} f$. From (6) we obtain

$$
\sum_{i=1}^{3} g_{\imath}=1 \text {. }
$$

By Lemma 3 we know that $g_{1}, g_{2}$ and $g_{3}$ are linearly independent. In a similar manner we get

$$
T(r, g)<N\left(r, \frac{1}{f}\right)+N\left(r, \frac{1}{g}\right)+2 \bar{N}(r, f)+o(T(r)) \quad(r \notin E) .
$$

From (12) and (13) we deduce

$$
T(r)<N\left(r, \frac{1}{f}\right)+N\left(r, \frac{1}{g}\right)+2 \bar{N}(r, f)+o(T(r)) \quad(r \notin E) .
$$

Combining (2) and (14) we get

which is impossible.

$$
(1-\mu) T(r)<o(T(r)) \quad(r \in I),
$$

b) Suppose that $f_{1}, f_{2}$ and $f_{3}$ are linearly dependent. Then, there exist three constants $\left(c_{1}, c_{2}, c_{3}\right) \neq(0,0,0)$ such that

$$
c_{1} f_{1}+c_{2} f_{2}+c_{3} f_{3}=0 \text {. }
$$

If $c_{1}=0$, from (16) we have $c_{2} \neq 0, c_{3} \neq 0$ and

$$
f_{3}=-\frac{c_{2}}{c_{3}} f_{2}
$$

and hence

$$
g=\frac{c_{2}}{c_{3}},
$$

which is impossible. Thus $c_{1} \neq 0$ and

$$
f_{1}=-\frac{c_{2}}{c_{1}} f_{2}-\frac{c_{3}}{c_{1}} f_{3} .
$$

Now combining (6) and (17) we get

$$
\left(1-\frac{c_{2}}{c_{1}}\right) f_{2}+\left(1-\frac{c_{3}}{c_{1}}\right) f_{3}=1 \text {. }
$$

We discuss the following three subcases.

$b_{1}$ ) Assume $c_{1}=c_{2}$. From (18) we have $c_{1} \neq c_{3}$ and

$$
f_{3}=\frac{c_{1}}{c_{1}-c_{3}},
$$


that is

$$
g=-\frac{c_{1}}{c_{1}-c_{3}} e^{-n}
$$

From (6) and (19) we get

$$
f_{1}+f_{2}=-\frac{c_{3}}{c_{1}-c_{3}}
$$

that is

$$
f+e^{h}=-\frac{c_{3}}{c_{1}-c_{3}} .
$$

If $c_{3} \neq 0$, from $(20)$ and $(21)$ we have

$$
T(r)=T\left(r, e^{h}\right)+O(1)
$$

and

$$
N\left(r, \frac{1}{f}\right)+N\left(r, \frac{1}{g}\right)+2 \bar{N}(r, f)=T\left(r, e^{h}\right)+S(r, f)=(1+o(1)) T(r) \quad(r \notin E),
$$

which contradicts our assumption. Thus $c_{3}=0$. From (20) and (21) we deduce $g=-e^{-h}$ and $f=-e^{h}$ and hance $f \cdot g=1$.

$b_{2}$ ) Assume $c_{1}=c_{3}$. From (18) we have $c_{1} \neq c_{2}$ and

$$
f_{2}=\frac{c_{1}}{c_{1}-c_{2}}
$$

that is

$$
e^{h}=\frac{c_{1}}{c_{1}-c_{2}}
$$

From (6) and (22) we get

$$
f-\frac{c_{1}}{c_{1}-c_{2}} g=-\frac{c_{2}}{c_{1}-c_{2}} .
$$

If $c_{2} \neq 0$, by Lemma 1 we have

$$
T(r)<N\left(r, \frac{1}{f}\right)+N\left(r, \frac{1}{g}\right)+\bar{N}(r, f)+S(r, f) .
$$

By (2) and (24) we get

$$
(1-\mu) T(r)<o(T(r)) \quad(r \in I),
$$

which is impossible. Thus $c_{2}=0$. From (23) we deduce $f=g$.

$\mathrm{b}_{3}$ ) Assume $c_{1} \neq c_{2}$ and $c_{1} \neq c_{3}$. From (18) we have

$$
g=\frac{c_{1}-c_{2}}{c_{1}-c_{3}}-\frac{c_{1}}{c_{1}-c_{3}} e^{-h}
$$

Now combining (17) and (26), we get 


$$
f=-\frac{c_{2}-c_{3}}{c_{1}-c_{3}} e^{h}-\frac{c_{3}}{c_{1}-c_{3}} .
$$

From (26) and (27) we have

$$
T(r)=T\left(r, e^{n}\right)+O(1)
$$

and

$$
N\left(r, \frac{1}{g}\right)=T\left(r, e^{h}\right)+S(r, g)=(1+o(1)) T(r) \quad(r \notin E),
$$

which contradicts our assumption.

This completes the proof of Theorem 2 .

\section{Proof of Theorem 1 .}

Suppose that $f \neq g$. By assumption we have with two entire functions $\alpha$ and $\beta$,

$$
f=e^{\alpha} \cdot g, \quad f-1=e^{\beta} \cdot(g-1) .
$$

Since $f \neq g$, then $e^{\beta} \neq 1$ and $e^{\beta-\alpha} \neq 1$. Thus from (28) we get

$$
f=\frac{1-e^{\beta}}{1-e^{\beta-\alpha}}
$$

and

$$
T\left(r, e^{\alpha}\right)+T\left(r, e^{\beta}\right)=O(T(r)) .
$$

If $e^{\beta}=c$, where $c(\neq 0,1)$ is a constant, then from (29) we have

$$
N\left(r, \frac{1}{f}\right)=0 \text {. }
$$

If $e^{\beta}$ is not a constant, let $\left\{z_{n}\right\}$ be all the roots of $f=0$ with multiplicity $\geqq 2$, then from (29) $\left\{z_{n}\right\}$ are the roots of $\left(1-e^{\beta}\right)^{\prime}=-\beta^{\prime} e^{\beta}=0$. Thus

$$
N\left(r, \frac{1}{f}\right)-N_{1}\left(r, \frac{1}{f}\right) \leqq 2 N\left(r, \frac{1}{\beta^{\prime}}\right) \leqq 2 T\left(r, \beta^{\prime}\right)+O(1) .
$$

By Lemma 4 and (30) we have

$$
N\left(r, \frac{1}{f}\right) \leqq N_{1}\left(r, \frac{1}{f}\right)+o(T(r)) \quad(r \notin E) .
$$

If $e^{\beta-\alpha}=c(\neq 0,1)$, then from (29) we have

$$
N(r, f)=0 .
$$

If $e^{\beta-\alpha}$ is not a constant, let $\left\{t_{n}\right\}$ be all the roots of $1 / f=0$ with multiplicity $\geqq 2$, then from (29) $\left\{t_{n}\right\}$ are the roots of $\left(1-e^{\beta-\alpha}\right)^{\prime}=-\left(\beta^{\prime}-\alpha^{\prime}\right) e^{\beta-\alpha}=0$. Thus 


$$
N(r, f)-N_{1}(r, f) \leqq 2 N\left(r, \frac{1}{\beta^{\prime}-\alpha^{\prime}}\right) \leqq 2 T\left(r, \alpha^{\prime}\right)+2 T\left(r, \beta^{\prime}\right)+O(1) .
$$

By Lemma 4 and (30) we have

$$
N(r, f) \leqq N_{1}(r, f)+o(T(r)) \quad(r \notin E) .
$$

Noting $N(r, 1 / g)=N(r, 1 / f)$ and $N(r, g)=N(r, f)$, from (31), (32), (33) and (34) we deduce

$$
N\left(r, \frac{1}{f}\right)+N\left(r, \frac{1}{g}\right)+2 \bar{N}(r, f)<2 N_{1}\left(r, \frac{1}{f}\right)+2 N_{1}(r, f)+o(T(r)) \quad(r \notin E) .
$$

Now combining (1) and (35) we obtain

$$
N\left(r, \frac{1}{f}\right)+N\left(r, \frac{1}{g}\right)+2 \bar{N}(r, f)<(2 \lambda+o(1)) T(r) \quad(r \in I) .
$$

By Theorem 2 we deduce the conclusion of Theorem 1 .

\section{An Application of Theorem 1.}

Let $f$ be a nonconstant meromorphic function and $S$ be a set in the complex plane, and let

$$
E_{f}(S)=\bigcup_{a \in S}\{z \mid f(z)-a=0\}
$$

where any $z$ which is a zero of multiplicity $m$ is included in $E_{f}(S), m$ times.

In [1] F. Gross and C.F. Osgood proved the following theorem.

THEOREM G. Let $S_{1}=\{-1,1\}, S_{2}=\{0\}$. If $f$ and $g$ are entire functions of finite order such that $E_{f}\left(S_{\imath}\right)=E_{g}\left(S_{\imath}\right)(i=1,2)$, then $f= \pm g$ or $f \cdot g= \pm 1$.

In [10] the present author proved that in the preceding theorem the order restriction of $f$ and $g$ can be removed. The present author [12] and independently $\mathrm{K}$. Tohge [6] proved the following result which is an extension of the above results.

THEOREM H. Let $S_{1}=\left\{1, \omega, \cdots, \omega^{n-1}\right\}, S_{2}=\{0\}$ and $S_{3}=\{\infty\}$, where $n$ is an integer $(\geqq 2)$ and $\omega=\cos (2 \pi / n)+i \sin (2 \pi / n)$. If $f$ and $g$ are meromorphic functions such that $E_{f}\left(S_{\imath}\right)=E_{g}\left(S_{\imath}\right)(i=1,2,3)$, then $f^{n}=g^{n}$ or $f^{n} \cdot g^{n}=1$.

Using Theorem 1 , it is easy to give the proof of Theorem $\mathrm{H}$. In fact, let $F=f^{n}$ and $G=g^{n}$, then $\boldsymbol{F}$ and $\boldsymbol{G}$ share $0,1, \infty C M$ and $N_{1}(r, 1 / F)+N_{1}(r, F)=0$. By Theorem 1 , we get $F=G$ or $F \cdot G=1$, that is $f^{n}=g^{n}$ or $f^{n} \cdot g^{n}=1$. This proves Theorem $\mathrm{H}$.

Acknowledement. I am grateful to the referee for valuable comments. 


\section{REFERENCES}

[1] F. Gross AND C.F. OsGood, Entire functions with common preimages, Factorization Theory of Meromorphic Functions, 19-24, Marcel Dekker, Inc., 1982.

[2] G.G. Gundersen, Meromorphic functions that share three or four values, J. London Math. Soc., (2), 20 (1979), 457-466.

[3] W.K. Hayman, Meromorphic Functions, Clarendon Press, Oxford, 1964.

[4] R. Nevanlinna, Le Théorèms de Picard-Borel et la Théorie des Fonctions Méromorphes, Gauthier-Villars, Paris, 1929.

[5] M. Ozawa, Unicity theorems for entire functions, J. d'Anal. Math., 30 (1976), $411-420$.

[6] K. TOHGE, Meromorphic functions covering certain finite sets at the same points, Kodai Math. J., 11 (1988), 249-279.

[ 7 ] H. UEDA, Unicity theorems for entire functions, Kodai Math. J., 3 (1980), 212-223.

[8] H. UEDA, Unicity theorems for meromorphic or entire functions, Kodai Math. J., 3 (1980), 457-471.

[9] H. UEDA, Unicity theorems for meromorphic or entire functions II, Kodai Math. J., 6 (1983), 26-36.

[10] HoNG-XUN YI, Meromorphic functions with common premages, J. of Math. (PRC), 7 (1987), 219-224.

[11] Hong-XUn YI, Meromorphic functions with two deficient values, Acta Math. Sin., 30 (1987), 588-597.

[12] Hong-XUn YI, On the uniqueness of meromorphic functions, Acta Math. Sin., 31 (1988), 570-576.

[13] Hong-XUN YI, Meromorphic functions that share three values, Chin. Ann. Math., 9A (1988), 434-440.

Department of Mathematics

SHANDONG UNIVERSITY

JinAN, SHANDONG, 250100

P.R. CHINA 